\title{
Systematic review of stimulant and nonstimulant laxatives for the treatment of functional constipation
}

\author{
Pierre Paré $M D^{1}$, Richard N Fedorak MD²
}

\begin{abstract}
P Paré, RN Fedorak. Systematic review of stimulant and nonstimulant laxatives for the treatment of functional constipation. Can J Gastroenterol Hepatol 2014;28(10):549-557.
\end{abstract}

BACKGROUND: Constipation is an uncomfortable and common condition that affects many, irrespective of age. Since 1500 BC and before, health care practitioners have provided treatments and prevention strategies to patients for chronic constipation despite the significant variation in both medical and personal perceptions of the condition. OBJECTIVE: To review relevant research evidence from clinical studies investigating the efficacy and safety of commercially available pharmacological laxatives in Canada, with emphasis on studies adopting the Rome criteria for defining functional constipation.

SEARCH METHODS: PubMed, Medline, Embase and Evidence-Based Medicine Reviews databases were searched for blinded or randomized clinical trials and meta-analyses assessing the efficacy of nonstimulant and stimulant laxatives for the treatment of functional constipation.

RESULTS: A total of 19 clinical studies and four meta-analyses were retrieved and abstracted regarding study design, participants, interventions and outcomes. The majority of studies focused on polyethylene glycol compared with placebo. Both nonstimulant and stimulant laxatives provided better relief of constipation symptoms than placebo according to both objective and subjective measures. Only one study compared the efficacy of a nonstimulant versus a stimulant laxative, while only two reported changes in quality of life. All studies reported minor side effects due to laxative use, regardless of treatment duration, which ranged from one week to one year. Laxatives were well tolerated by both adults and children.

Key Words: Canadian Digestive Health Foundation (CDHF); Constipation; Fecal impaction; Nonstimulant laxative; Polyethylene glycol (PEG); Stimulant laxative

\section{L'analyse systématique de laxatifs stimulants et non stimulants pour traiter la constipation fonctionnelle}

HISTORIQUE : La constipation est un problème désagréable très répandu, quel que soit l'âge. Depuis 1500 av. J.-C. et même auparavant, les dispensateurs de soins ont proposé des traitements et des stratégies préventives aux patients pour soulager la constipation chronique, malgré l'importante variation entre les perceptions médicales et les perceptions personnelles à cet égard.

OBJECTIF : Analyser les données de recherche pertinentes tirées d'études cliniques sur l'efficacité et l'innocuité des laxatifs pharmacologiques sur le marché canadien, en s'attardant aux études ayant défini la constipation fonctionnelle selon le critère de Rome.

MÉTHODOLOGIE : Les chercheurs ont fouillé les bases de données de PubMed, Medline, Embase et Evidence-Based Medicine Reviews pour en extraire des essais cliniques aléatoires ou en insu et des métaanalyses sur l'efficacité des laxatifs non stimulants et stimulants pour traiter la constipation fonctionnelle.

RÉSULTATS : Au total, 19 études cliniques et quatre méta-analyses ont été extraites. On en a résumé la méthodologie, le type de participants, les interventions et les résultats. La majorité des études portaient sur le polyéthylène glycol comparé à un placebo. Que les mesures soient objectives ou subjectives, tant les laxatifs non stimulants que stimulants soulageaient davantage les symptômes de constipation qu'un placebo. Une seule étude comparait l'efficacité d'un laxatif non stimulant à un laxatif stimulant, tandis que deux seulement faisaient état de changements à la qualité de vie. Toutes les études signalaient des effets secondaires mineurs causés par les laxatifs, quelle que soit la durée du traitement, qui variait entre de une semaine et un an. À la fois les adultes et les enfants toléraient les laxatifs.

Initiated by the Rome Foundation, the first comprehensive, consensus-driven, multinational diagnostic guidelines for constipation were presented as 'Rome II' in 1999 and have since been updated by the more expansive 'Rome III' (4,5). Functional constipation describes episodic constipation in the absence of any physiological abnormalities, such as pelvic organ prolapse or obstruction, and the symptoms do not fulfill the diagnostic criteria of irritable bowel syndrome, subtype: constipation (Table 1) (4). Specifically, constipation associated with anorectal dysfunction is covered by unique diagnostic criteria in Rome III (4).

Due to the changing medical definition of constipation pre-Rome III (4) and patient perceptions, it is difficult to determine changes in incidence and prevalence over time. For example, of 220 Canadian patients who complained of constipation or had received a diagnosis of it, only $37.3 \%$ fulfilled the Rome II diagnostic criteria (6). Nevertheless, a Canadian survey-based study found that the prevalence of functional constipation (Rome II) was $14.9 \%$, which was comparable with the $19.9 \%$ rate previously reported for Olmsted County in the United States $(7,8)$. Based on consumer demand, patients and their clinicians.

${ }^{1} \mathrm{CHU}$ de Quebec - Hôpital du Saint-Sacrement, Université Laval, Quebec City, Quebec; ${ }^{2}$ Division of Gastroenterology, University of Alberta, Edmonton, Alberta

Correspondence: Dr Richard N Fedorak, University of Alberta, 2-14A Zeidler Ledcor Centre, Edmonton, Alberta T6G 2 X8.

Telephone 780-492-6941, fax 780-492-8121, e-mail richard.fedorak@ualberta.ca

Received for publication August 7, 2014. Accepted September 17, 2014 
TABLE 1

The Rome III diagnostic criteria* for functional constipation and irritable bowel syndrome (IBS) (4)

\begin{tabular}{|c|c|}
\hline Functional constipation & IBS \\
\hline \multirow{2}{*}{$\begin{array}{l}\text { 1. Must include two or more of the following: } \\
\text { a) Straining during at least } 25 \% \text { of defecations }\end{array}$} & Recurrent abdominal pain or discomfort ${ }^{\dagger}$ for at least three days per month in the \\
\hline & past three months associated with two or more of the following: \\
\hline b) Lumpy or hard stools in at least $25 \%$ of defecations & 1. Improvement with defecation \\
\hline d) Sensation of anorectal obstruction/blockage for at least $25 \%$ of defecations & 3. Onset associated with a change in form (appearance) of stool \\
\hline \multirow{2}{*}{$\begin{array}{l}\text { e) Manual manoeuvres to facilitate at least } 25 \% \text { of defecations (eg, digital } \\
\text { evacuation, support of the pelvic floor) }\end{array}$} & IBS with constipation subtype: \\
\hline & Hard or lumpy stools $\geq 25 \%$ and loose (mushy) or watery stools $<25 \%$ of \\
\hline 2. Loose stools are rarely present without the use of laxatives & Scale \\
\hline 3. Insufficient criteria for IBS & \\
\hline
\end{tabular}

${ }^{*}$ Criteria fulfilled for the past three months with symptom onset at least six months before diagnosis; ${ }^{\dagger}$ Discomfort means an uncomfortable sensation not described as pain

Canada is the ninth largest market in the world for over-the-counter laxatives (9). Since 2003, the annual spending increase in the Canadian market is US\$7.4 million (9).

Certain risk factors are known to predispose individuals to functional constipation including sex, increasing age and socioeconomic situation (7,10-13). The economic burden of constipation, perceived or fulfilling Rome III criteria, is substantial, with one-third of Canadian sufferers seeking medical attention (12). Patients have a significantly impaired health-related quality of life that improves on relief of their constipation (12).

If left untreated, chronic constipation may progress to fecal impaction, in which the rectum becomes obstructed with immobile fecal matter. Treatment frequently entails manual disimpaction. Even the Ebers medical Papyrus from Egypt, dating to circa 1550 BC, provides a long list of laxatives specific for adults and children (14). Instead of laxatives, both the Rome III and the 2010 World Gastroenterology Organisation Guidelines on Constipation recommend that the patient stop any constipating medication if possible, treat depression and hypothyroidism if present, and increase dietary fibre intake $(4,15)$. If these measures fail, then it is recommended to use laxatives. Surgical intervention is a last resort.

In general, laxatives can be divided into two categories: nonstimulant or osmotic, and stimulant laxatives. Over the past 30 years, stimulant laxatives fell out of favour in preference for nonstimulants (16). In the absence of long-term or placebo-controlled clinical studies, initial observations by Smith $(17,18)$ - that long-term use of stimulant laxatives damaged the enteric nervous system - prevailed. However, current biological evidence and clinical guidelines indicate that stimulants are both effective and safe for the treatment of functional constipation (19-21). To this end, we conducted a systematic literature review and present the evidence.

\section{METHODS}

The PubMed, Medline, Embase and Evidence-Based Medicine Reviews (including the Cochrane Library) databases were searched using the terms "laxative" and "constipation" as key words and medical subject headings. Generic names of laxatives were also searched (eg, "milk of magnesia" and "docusate sodium"). Restrictions imposed were English language and human subjects; study design was limited to clinical trial (all), systematic review (for pearling purposes) or metaanalysis. Each database was searched from the time of its inception until January 30, 2014.

During in-depth review of each article, only pharmacological laxative studies that were blinded randomized controlled trials (RCTs) were retained for data abstraction. Studies assessing laxative efficacy in subjects who had known health conditions or were receiving medical treatments causing or associated with constipation were excluded. Additionally, all studies assessing the efficacy of laxatives for the purpose of bowel or abdominal imaging or surgery were excluded.
Although tegaserod studies were retrieved during the search, they were excluded from data abstraction in light of the suspension imposed on its marketing and sales by both Health Canada and the United States Food and Drug Administration.

\section{Nonstimulant laxatives}

\section{RESULTS}

Stool softeners: Only one study examining the efficacy of the stool softener docusate sodium was identified that fulfilled the selection criteria. In this multicentre, double-blinded RCT, patients $(n=170)$ with chronic idiopathic constipation, including nonproductive bowel movement criteria, received either docusate sodium (Colace [Purdue Pharma LP, USA], $100 \mathrm{mg}$ twice per day) or psyllium (Metamucil [Procter \& Gamble, USA], $5.1 \mathrm{~g}$ twice per day) for two weeks following a one-week washout and one-week baseline period. In week 2, the docusate sodium treatment led to a mean of 2.9 bowel movements per week compared with 3.5 for psyllium $(\mathrm{P}=0.02)$. It is important to note that the baseline bowel movement means for docusate sodium and psyllium were 3.4 and 3.1 per week, respectively, suggesting that docusate sodium appears to decrease the weekly bowel movement frequency. Psyllium treatment was also significantly better for increasing stool water content, stool water weight, total stool output and O'Brien rank-type score, which combines objective measures of constipation (22). No adverse events were reported.

Emollients and lubricants: Two studies examined liquid paraffin, an emollient, compared with either lactulose or polyethylene glycol (PEG) in pediatric patients. In the first study, 40 children (mean age 3.7 years), who had $<3$ bowel movements per week, painful defecation, rectal bleeding and encopresis for $>3$ months, were randomly assigned $1: 1$ to receive open-label liquid paraffin or lactulose (23). Urganci et al (23) found that paraffin was significantly better than lactulose with respect to stool consistency at week $4(\mathrm{P}<0.01)$, although there was no significant difference at week 8 . Baseline values of 1.9 bowel movements per week increased to 16.1 for liquid paraffin and 12.3 for lactulose after eight weeks $(\mathrm{P}<0.05)$. Patient compliance was significantly different in week 8 , with $90 \%$ of patients taking liquid paraffin versus only $60 \%$ of the lactulose group $(\mathrm{P}=0.02)$.

Rafati et al (24) enrolled 158 patients (mean age 51 months) who had functional constipation defined as $<3$ stools per week, $>1$ encopresis per week, or palpable abdominal or rectal fecal mass. After randomization to either liquid paraffin or PEG 3350 for four months, the mean number of bowel movements per week increased from a baseline of 1.5 to 7.5 , and 8.7 per week for liquid paraffin and PEG, respectively $(\mathrm{P}=0.58)$. The mean number of encopresis events decreased from baseline; however, no significant difference between the two groups was reported at the conclusion of the study. However, because there were significantly more adverse events with paraffin, the authors recommended PEG 3350 treatment for constipation. In consideration of the longer duration of the study by Rafati et al (24) (four months), it may 
be that liquid paraffin is suitable for use over a short period (eg, eight weeks); however, PEG may be more beneficial for those who require a longer treatment window. It should be noted that 82 study participants received bisacodyl suppositories for fecal impaction at the onset of the study. This dual therapy may have contributed to the incidence of adverse events; however, the authors did not discuss this nor provide details regarding the timeline of adverse events.

\section{Osmotic agents}

A total of 20 original studies and four meta-analyses assessing osmotic laxatives were retrieved, of which 19 had PEG with or without electrolytes as a treatment arm (Table 2).

PEG without electrolytes versus placebo: In the four retrieved studies that investigated PEG without electrolytes versus placebo, the mean number of bowel movements per week were significantly higher in the treatment groups; subjective measures were also more improved with PEG (25-28). The largest $(n=304)$ and longest (six months) study was conducted by DiPalma et al (27) in 2007, and was also the only one that used the Rome III diagnosis of functional constipation. This double-blinded trial, conducted at 50 centres, randomly assigned patients to PEG $(n=204)$ or placebo $(n=100)$ treatment. At the conclusion of the study, the mean number of bowel movements per week was 7.9 versus 5.6 for $\mathrm{PEG}$ and placebo groups, respectively $(\mathrm{P}<0.001)$. Although there was no significant difference with respect to adverse events, the trend was that PEG treatment resulted in more flatulence, diarrhea and nausea than placebo. However, $52 \%$ of patients found that PEG was successful for achieving $\geq 3$ bowel movements per week with ease compared with only $11 \%$ of patients receiving placebo $(\mathrm{P}<0.001)$.

Nurko et al (28) conducted a two-week double-blind RCT involving 103 pediatric patients (mean age 8.5 years). They also found that PEG significantly improved the frequency of weekly bowel movements compared with placebo $(P<0.001)$, the number of patients who had $\geq 3$ bowel movements per week $(\mathrm{P}<0.04)$ and overall reduction in straining $(\mathrm{P}<0.05)$. In this dosing study, both stool consistency and fewer incidents of abdominal pain or fecal incontinence were reported for PEG at $0.4 \mathrm{~g} / \mathrm{kg} /$ day than at $0.8 \mathrm{~g} / \mathrm{kg} /$ day.

PEG with electrolytes versus placebo: The efficacy of PEG with electrolytes versus placebo was investigated in three studies, while another explored the effectiveness against baseline (29-31). The only double-blinded RCT was conducted by Corazziari et al (30) at six centres. Recruitment was restricted to adults (mean age 42 years) who had $<2$ bowel movements per week for $\geq 12$ months, or at least two of the following in the absence of treatment: $<3$ bowel movements per week, straining, sensation of incomplete evacuation and hard stools $>25 \%$ of the time. Following a four-week evaluation period, during which laxative use was restricted, constipated patients were then randomly assigned to either PEG plus electrolytes $(n=25)$ or placebo $(n=23)$ for eight weeks. At the conclusion of the study, PEG treatment was significantly better than placebo for stool frequency, consistency and decreased straining. Similar findings were reported in the cross-over study for both objective and subjective measures for adults $(29,31)$.

PEG versus placebo: In two of the meta-analyses retrieved, PEG with or without electrolytes was compared with placebo (32,33). In 2010, Belsey et al (32) analyzed the pooled data from 10 studies and found that PEG was significantly more effective than placebo with respect to the mean frequency of stools per week (mean deviation [MD] $=1.98$, $\mathrm{P}=0.0003$ ). Selecting seven of the higher-quality studies, the mean number of stools per week in PEG-treated patients increased to an MD of 2.43 compared with placebo ( $\mathrm{P}=0.0001)$. In 2012, Gordon et al (33) selected two PEG pediatric studies and similarly found that the MD of 2.61 stools per week favoured PEG over placebo (33).

PEG 4000 with electrolytes versus PEG 3350 without electrolytes: Two studies comparing PEG with and without electrolytes were retrieved. From 69 centres in France, adults (mean age 52 years) who had a history of constipation for at least three months and one of the following were recruited: $<3$ bowel movements per week, hard/lumpy stools associated with straining or a feeling of incomplete evacuation (34). To rule out physical causes of constipation, all patients had to have undergone either a colonoscopy or barium enema in the five years before the study. Patients $(n=270)$ were randomly assigned to either PEG with electrolytes (Transipeg [Bayer Consuemer Care AG, Switzerland] $5.9 \mathrm{~g} /$ day or $11.8 \mathrm{~g} /$ day) or without (Forlax [Ipsen, France] $10 \mathrm{~g} /$ day or $20 \mathrm{~g} /$ day). After a one-month study period, the mean number of bowel movements per week and stool consistency increased significantly compared with baseline in each treatment arm $(\mathrm{P}=0.0001$ for all). The only notable difference was a trend indicating that the standard dose of PEG with electrolytes provided more patients with stools of normal consistency compared with those taking PEG without electrolytes at the standard dose.

In 2012, a study also investigated PEG with and without electrolytes using a cohort of 49 children two to 16 years of age who had Rome III defined functional constipation or fecal impaction (35). The 42 children receiving PEG with electrolytes (Movicol [Norgine, United Kingdom]) had significantly fewer bowel movements per week than the 49 patients receiving PEG (7.8 versus 9.2; $\mathrm{P}=0.025)$. Interestingly, $52 \%$ of patients taking PEG with electrolytes did not experience difficulties taking the medication compared with $96 \%$ taking PEG $(\mathrm{P}<0.001)$, presumably mediated by the better taste of PEG $(\mathrm{P}<0.001)$. The palatability of an oral treatment for use in the longterm is of key concern because it may impact patient compliance.

By examining the role of PEG in the management of functional constipation, defined by Rome III or otherwise, highlights that PEG is significantly more effective than placebo for increasing bowel movement frequency and stool consistency, improving subjective measures, and is not associated with adverse events in either short- or long-term studies of various designs. These findings are applicable to both adult and pediatric patients. Although the use of PEG with or without electrolytes appears to offer similar efficacy, the palatability of PEG without electrolytes is preferential to recommend to ensure high rates of compliance.

Osmotic agents, in general, versus placebo: In a 2011 meta-analysis, Ford and Suares (36) examined the efficacy of osmotic laxatives (PEG with or without electrolytes and lactulose) versus placebo. Pooling data from five studies, $37.2 \%$ of those who took osmotic laxatives were nonresponders compared with $68.9 \%$ who took placebo; the number needed to treat was 3 ( $95 \%$ CI 2 to 4). Using data from six studies, the weighted mean difference between the mean number of stools per week for osmotic laxatives versus placebo was 2.51 (95\% CI 1.30 to 3.71). Thus, based on objective measures, osmotic agents provided improved resolution of functional constipation.

PEG compared with lactulose: The selection criteria in the present analysis identified five articles reporting findings from RCTs ranging in length from four weeks to three months, involving both adults and children. At best, two studies found no significant difference between PEG and lactulose with respect to the mean number of bowel movements per week $(37,38)$. With respect to subjective measures, only one study reported that patients receiving PEG had $60 \%$ of their stools as soft or liquid compared with $40 \%$ in the lactulose group (37). Conversely, in two studies, PEG significantly improved the mean number of bowel movements per week compared with lactulose $(39,40)$. A third study reported significantly more patients achieving treatment success, defined as having $\geq 3$ bowel movements per week and $\leq 1$ encopresis episode(s) per week with PEG compared with lactulose (41). Two studies found that PEG treatment led to significantly fewer fecal impactions and reduced the need for other laxatives $(38,40)$.

Furthermore, each of the three meta-analyses retrieved found that PEG was superior to lactulose for the treatment of constipation based on the mean difference in the number of stools per week between the treatment groups $(32,33,42)$. The meta-analysis by Lee-Robichaud et al (42) also determined that PEG was significantly better than lactulose with respect to Bristol stool score (pooled results from two studies), relief of abdominal pain (pooled results from two studies) and use of additional laxatives (three studies). 
TABLE 2

Summary of studies examining the efficacy of nonstimulant laxatives

\begin{tabular}{|c|c|c|c|c|c|}
\hline $\begin{array}{l}\text { Author (ref), } \\
\text { year }\end{array}$ & Design/duration & $\begin{array}{l}\text { Treatment } \\
\text { (n, mean age) }\end{array}$ & $\begin{array}{l}\text { Comparator } \\
\text { (n, mean age) }\end{array}$ & Measures & Outcomes \\
\hline $\begin{array}{l}\text { Cleveland et al } \\
\text { (25), } 2001\end{array}$ & $\begin{array}{l}\text { Double-blinded, random- } \\
\text { ized crossover trial } \\
\text { (14 days }+14 \text { days })\end{array}$ & $\begin{array}{l}\text { PEG }(n=23 \\
47.7 \text { years })\end{array}$ & Placebo & Mean BM/week & PEG (7.0) vs placebo (3.6); $P=0.0001$ \\
\hline \multirow[t]{2}{*}{$\begin{array}{l}\text { DiPalma et al } \\
(26), 2000\end{array}$} & $\begin{array}{l}\text { Blinded RCT at four } \\
\text { centres }\end{array}$ & $\begin{array}{l}\text { PEG }(17 \text { g/day })(n=80 \\
46.7 \text { years })\end{array}$ & $\begin{array}{l}\text { Placebo }(n=71 \\
45.8 \text { years })\end{array}$ & Mean BM/week & PEG (4.5) vs placebo (2.7); $P<0.01$ \\
\hline & 2 weeks & & & Subjective measures & $\begin{array}{l}\text { PEG was preferred by investigators } \\
(P<0.005) \text { and patients }(P<0.001) \text {. }\end{array}$ \\
\hline \multirow[t]{3}{*}{$\begin{array}{l}\text { DiPalma et al } \\
\text { (27), 2007, } \\
\text { Rome III }\end{array}$} & $\begin{array}{l}\text { Double-blinded RCT in } \\
50 \text { centres }\end{array}$ & $\begin{array}{l}\text { PEG } 335017 \mathrm{~g} / \text { day } \\
(n=204,53.1 \text { years })\end{array}$ & $\begin{array}{l}\text { Placebo }(n=100 \\
54.4 \text { years })\end{array}$ & Subjective measure - success & $\begin{array}{l}52 \% \text { of } P E G \text { vs } 11 \% \text { of placebo rated } \\
\geq 50 \% \text { of the treatment weeks as } \\
\text { successful ( } \geq 3 \mathrm{BM} / \text { week, and ease); } \\
P<0.001\end{array}$ \\
\hline & 6 months & & & Mean BM/week & PEG > Placebo (7.9 vs 5.6); $P<0.001$ \\
\hline & & & & Rescue meds/week & PEG < Placebo (2.8 vs 3.9); P<0.138 \\
\hline \multirow[t]{3}{*}{$\begin{array}{l}\text { Nurko et al } \\
(28), 2008\end{array}$} & Double-blinded RCT & $\begin{array}{l}\text { PEG } 3350 \text { (0.2, } 0.4 \text { or } \\
0.8 \mathrm{~g} / \mathrm{kg} / \mathrm{day})\end{array}$ & $\begin{array}{l}\text { Placebo }(n=24 \\
8.0 \text { years })\end{array}$ & $\geq 3 \mathrm{BM} /$ week & $\begin{array}{l}\text { PEG > Placebo }(P<0.04 \text { for each study } \\
\text { group). NSD between each } P E G \text { group }\end{array}$ \\
\hline & 2 weeks & $\begin{array}{c}n=26,27 \text { or } 26(8.6 \\
9.1 \text { or } 8.4 \text { years })\end{array}$ & & Mean BM/week & PEG > Placebo; $P<0.001$ \\
\hline & & & & Subjective measures & $\begin{array}{c}\text { Straining: } P E G>\text { Placebo }(P<0.05) \text {. } \\
\text { Stool consistency was better at } \\
0.4 \text { and } 0.8 \mathrm{~g} / \mathrm{kg} / \text { day; } P<0.001\end{array}$ \\
\hline \multirow[t]{2}{*}{$\begin{array}{l}\text { Andorsky and } \\
\text { Goldner (29), } \\
1990\end{array}$} & $\begin{array}{l}\text { Controlled, double- } \\
\text { blinded, randomized, } \\
\text { cross-over ( } 5 \text { days + } \\
5 \text { days) }\end{array}$ & $\begin{array}{l}\text { PEG+E ( } 8 \text { or } 16 \text { oz/ } \\
\text { day) }(n=32, n / a)\end{array}$ & $\begin{array}{l}\text { Placebo (8 or } \\
16 \text { oz/day) }\end{array}$ & Mean BM/week & $\begin{array}{l}\mathrm{PEG}+\mathrm{E} 7.75 \pm 4.55 \text { vs placebo } \\
4.88 \pm 2.62 ; \mathrm{P}<0.01\end{array}$ \\
\hline & & & & Mean stool consistency & $\begin{array}{l}P E G+E 2.56 \pm 1.17 \text { vs placebo } \\
1.91 \pm 0.94 ; P<0.05\end{array}$ \\
\hline \multirow[t]{4}{*}{$\begin{array}{l}\text { Corazziari et al } \\
\text { (30), } 1996\end{array}$} & $\begin{array}{l}\text { Double-blinded RCT at } \\
6 \text { centres }\end{array}$ & $\begin{array}{l}\text { PEG+E as } 1 \text { sachet } \\
(17.5 \mathrm{~g}) \text { in } 250 \mathrm{~mL} \text { of } \\
\text { water twice per day } \\
(\mathrm{n}=25,40.3 \text { years })\end{array}$ & $\begin{array}{l}\text { Placebo }(n=23 \text {, } \\
43.5 \text { years })\end{array}$ & Mean BM/week & $\begin{array}{l}\text { PEG+E: } 4.8 \pm 2.3 \text { vs placebo } 2.8 \pm 1.6 \\
P<0.002\end{array}$ \\
\hline & 8 weeks & & & Straining & PEG+E > placebo; P<0.01 \\
\hline & & & & Stool consistency & $\mathrm{PEG}+\mathrm{E}>$ placebo; $\mathrm{P}<0.02$ \\
\hline & & & & Use of laxatives & $\mathrm{PEG}+\mathrm{E}>$ placebo; $\mathrm{P}<0.03$ \\
\hline \multirow[t]{2}{*}{$\begin{array}{l}\text { Thomson et al } \\
\text { (31), } 2007\end{array}$} & $\begin{array}{l}\text { Double-blinded ran- } \\
\text { domized crossover, } \\
6 \text { centres } 2 \text { weeks + } \\
2 \text { weeks washout + } \\
2 \text { weeks }\end{array}$ & $\begin{array}{l}\text { PEG } 3350+E(n=47 \\
2-11 \text { years })\end{array}$ & Placebo & Mean BM/week & $\begin{array}{l}\text { PEG+E > placebo for completed } \\
\text { defecations/week ( } 3.12 \text { vs } 1.45) \\
P<0.001\end{array}$ \\
\hline & & & & Subjective measures & $\begin{array}{l}\text { PEG+E was significantly better for: } \\
\text { straining, pain and stool consistency; } \\
P<0.05\end{array}$ \\
\hline \multirow[t]{2}{*}{$\begin{array}{r}\text { Belsey et al } \\
\text { (32), } 2010\end{array}$} & $\begin{array}{l}\text { Meta-analysis } \\
\text { PEG vs placebo: } \\
7 \text { high-quality studies }\end{array}$ & \multicolumn{2}{|c|}{ PEG, n=683; placebo, n=568 } & $\begin{array}{l}\text { Mean stools/week: } M D=2.34 \\
\text { stools/week favoured } P E G \\
(P=0.0001)\end{array}$ & $\begin{array}{l}\text { PEG was more effective than either } \\
\text { placebo or lactulose for treating } \\
\text { constipation }\end{array}$ \\
\hline & $\begin{array}{l}\text { PEG vs lactulose: } \\
4 \text { high-quality studies }\end{array}$ & \multicolumn{2}{|c|}{ PEG, $n=508$; lactulose, $n=451$} & $\begin{array}{l}\text { Mean stools/week: PEG } \\
M D=1.65 \text { stools/week } \\
(P=0.021)\end{array}$ & \\
\hline \multirow[t]{4}{*}{$\begin{array}{l}\text { Gordon et al } \\
\text { (33), } 2012\end{array}$} & $\begin{array}{l}\text { Meta-analysis } \\
18 \text { pediatric studies }\end{array}$ & \multicolumn{2}{|c|}{ PEG vs placebo, 2 studies, $n=101$} & $\begin{array}{l}\text { Mean stools/week: MD } \\
\text { favoured PEG at } 2.61 \text { stools/ } \\
\text { week }\end{array}$ & $\begin{array}{l}\text { The evidence quality was low or very } \\
\text { low for each finding and results should } \\
\text { be interpreted with caution }\end{array}$ \\
\hline & & \multicolumn{2}{|c|}{ PEG vs lactulose, 4 studies, $n=328$} & $\begin{array}{l}\text { Mean stools/week: MD } \\
\text { favoured PEG at } 1.09 \text { stools/ } \\
\text { week }\end{array}$ & \\
\hline & & \multicolumn{2}{|c|}{ PEG vs MOM, 3 studies, $n=211$} & $\begin{array}{l}\text { Mean stools/week: MD } \\
\text { favoured PEG at } 0.69 \text { stools/ } \\
\text { week }\end{array}$ & \\
\hline & & \multicolumn{2}{|c|}{ Liquid paraffin vs lactulose, 2 studies, $n=287$} & $\begin{array}{l}\text { Mean stools/week: MD } \\
\text { favoured paraffin at } \\
4.94 \text { stools/week }\end{array}$ & \\
\hline
\end{tabular}


TABLE 2 - CONTINUED

Summary of studies examining the efficacy of nonstimulant laxatives

\begin{tabular}{|c|c|c|c|c|c|}
\hline $\begin{array}{l}\text { Author (ref), } \\
\text { year }\end{array}$ & Design/duration & $\begin{array}{l}\text { Treatment ( } \mathrm{n} \text {, mean } \\
\text { age) }\end{array}$ & $\begin{array}{l}\text { Comparator ( } n \text {, mean } \\
\text { age) }\end{array}$ & Measures & Outcomes \\
\hline \multirow[t]{2}{*}{$\begin{array}{l}\text { Chaussade } \\
\text { and Minic } \\
(34), 2003\end{array}$} & $\begin{array}{l}\text { Double-blinded RCT in } \\
69 \text { centres }\end{array}$ & $\begin{array}{l}\text { PEG } 4000(10 \mathrm{~g} / \mathrm{day}) \\
(\mathrm{n}=66,50.6 \text { years })\end{array}$ & $\begin{array}{l}\text { PEG } 3350+E \\
(5.9 \text { g/day }) n=67 \\
50.2 \text { years })\end{array}$ & Mean BM/week & $\begin{array}{l}\text { All groups improved from baseline; } \\
\qquad=0.0001\end{array}$ \\
\hline & 4 weeks & $\begin{array}{l}\text { PEG } 4000(20 \text { g/day }) \\
(n=67,52.2 \text { years })\end{array}$ & $\begin{array}{l}\text { PEG } 3350+E \\
(11.8 \mathrm{~g} / \text { day }) \\
(\mathrm{n}=70,54.0 \text { years })\end{array}$ & Subjective measures & $\begin{array}{l}\text { All groups had improved measures; } \\
\qquad P=0.0001\end{array}$ \\
\hline \multirow{2}{*}{$\begin{array}{l}\text { Savino et al } \\
\text { (35), 2012, } \\
\text { Rome III or } \\
\text { fecal impac- } \\
\text { tion }\end{array}$} & Single blinded RCT & PEG ( $n=49,5.5$ years) & $\begin{array}{l}P E G+E(n=42 \\
5.6 \text { years })\end{array}$ & Mean stools/week & $\mathrm{PEG}>\mathrm{PEG}+\mathrm{E}$ (9.2 vs 7.8 ); $\mathrm{P}=0.025$ \\
\hline & & & & Subjective measures & $\begin{array}{l}\text { NSD between treatments for painful } \\
\text { stool, abdominal pain, soiling or use of } \\
\text { stimulants }\end{array}$ \\
\hline $\begin{array}{l}\text { Ford and } \\
\text { Suares (36), } \\
2011\end{array}$ & $\begin{array}{l}\text { Meta-analysis, } \\
6 \text { studies }\end{array}$ & $\begin{array}{l}\text { Osmotic laxatives, } \\
\text { n=396; placebo, } \\
\text { n=280 }\end{array}$ & & $\begin{array}{l}\text { Nonresponders (5/6 studies): } \\
\text { Osmotic laxatives, } 37.16 \% ; \\
\text { placebo, } 68.9 \% \text {. RR=0.50 } \\
\text { (95\% Cl } 0.39-0.63) \\
\text { Mean stools/week: The WMD } \\
\text { was } 2.51,(95 \% \mathrm{Cl} 1.30-3.71) \\
\text { favouring osmotic laxatives } \\
\text { NNT with osmotic laxatives = } 3 \\
\text { (95\% Cl } 2-4)\end{array}$ & $\begin{array}{l}\text { Nonstimulant laxatives were better than } \\
\text { placebo in the treatment of functional } \\
\text { constipation } \\
\text { Diarrhea was more common in } \\
\text { treatment groups than placebo } \\
\text { Difficulties experienced in comparing } \\
\text { treatment efficacy due to heterogeneity }\end{array}$ \\
\hline \multirow{2}{*}{$\begin{array}{l}\text { Bouhnik et al } \\
(37), 2004 \\
\text { Rome I }\end{array}$} & $\mathrm{RCT}$ & $\begin{array}{l}\text { Lactulose }(10-30 \mathrm{~g} / \mathrm{day}) \\
\qquad(\mathrm{n}=33,59 \text { years })\end{array}$ & $\begin{array}{l}\text { PEG } 4000(10-30 \text { g/day }) \\
\quad(n=32,57 \text { years })\end{array}$ & Mean BM/week & $\begin{array}{l}\text { Both groups had significant improvement } \\
\text { by } 62 \% \text { and } 66 \% \text {, respectively }\end{array}$ \\
\hline & 4 week & & & Subjective measures & $\begin{array}{l}\text { By week } 4,60 \% \text { of stools were either } \\
\text { soft or liquid with PEG vs } 40 \% \text { in } \\
\text { lactulose }\end{array}$ \\
\hline \multirow[t]{2}{*}{$\begin{array}{l}\text { Dupont et al } \\
(38), 2005\end{array}$} & Double-blinded RCT & $\begin{array}{l}\text { PEG (4-8 g/day) } \\
(n=51,28 \text { months })\end{array}$ & $\begin{array}{l}\text { Lactulose }(3.33- \\
6.66 \text { g/day })(n=45 \\
25.8 \text { months })\end{array}$ & Mean BM/week & $\begin{array}{l}\text { Significant improvements noted in both } \\
\text { groups in babies and toddlers }\end{array}$ \\
\hline & 3 months & & & Subjective measures & $\begin{array}{l}\text { PEG provided significantly improved } \\
\text { stool consistency, reduced use of other } \\
\text { laxatives, fecaloma and improved } \\
\text { appetite }\end{array}$ \\
\hline \multirow[t]{3}{*}{$\begin{array}{l}\text { Attar et al (39), } \\
1999\end{array}$} & $\begin{array}{l}\text { Blinded RCT at } \\
10 \text { centres }\end{array}$ & $\begin{array}{l}\text { PEG ( } 1 \text { sachet twice } \\
\text { per day) }(n=50 \\
55 \text { years) }\end{array}$ & $\begin{array}{l}\text { Lactulose biphar } \\
\qquad(1 \text { sachet twice per } \\
\text { day) ( } n=49 ; 55 \text { years) }\end{array}$ & Mean BM/day & $\begin{array}{l}\text { PEG }(1.3 \pm 0.7) \text { vs lactulose }(0.9 \pm 0.6) \\
P=0.005\end{array}$ \\
\hline & 4 weeks & & & Daily score for straining & $\begin{array}{l}\text { PEG }(0.5 \pm 0.7) \text { vs lactulose }(1.2 \pm 0.9) \\
P=0.0001\end{array}$ \\
\hline & & & & Overall improvement & $\begin{array}{l}\text { PEG }(7.4 \pm 2.5) \text { vs lactulose }(5.2 \pm 3.3) \\
\text { P<0.001 }\end{array}$ \\
\hline \multirow[t]{3}{*}{$\begin{array}{l}\text { Candy et al } \\
(40), 2006\end{array}$} & Double-blinded RCT & $P E G+E(n=27, n / a)$ & Lactulose $(n=26, n / a)$ & Mean BM/week & $\begin{array}{l}\text { PEG+E (9.4) vs lactulose (5.9); P=0.007 } \\
\text { at study end }\end{array}$ \\
\hline & 3 months & & & Reimpaction & PEG+E (0) vs lactulose (7); $P=0.011$ \\
\hline & & & & Rescue with senna & $\mathrm{PEG}+\mathrm{E}(0)$ vs lactulose (8); $P=0.002$ \\
\hline \multirow[t]{2}{*}{$\begin{array}{l}\text { Voskuijl et al } \\
\text { (41), } 2004\end{array}$} & $\begin{array}{l}\text { Double-blinded RCT } \\
8 \text { weeks }\end{array}$ & $\begin{array}{l}\text { PEG } 3350(n=46 \text {, } \\
6.5 \text { years })\end{array}$ & $\begin{array}{l}\text { Lactulose }(n=45 \text {, } \\
6.5 \text { years })\end{array}$ & Mean BM/week & $\begin{array}{l}\text { Improvement in both groups vs baseline; } \\
\mathrm{P}<0.01\end{array}$ \\
\hline & $\begin{array}{l}\text { Open-label for } \\
18 \text { weeks }\end{array}$ & & & Mean encopresis/week & $\begin{array}{l}\text { Improvement in both groups vs baseline; } \\
\mathrm{P}<0.01 \text {. NSD between treatments }\end{array}$ \\
\hline $\begin{array}{l}\text { Lee- } \\
\text { Robichaud } \\
\text { et al (42), } \\
2011\end{array}$ & $\begin{array}{l}\text { Meta-analysis } \\
8 \text { studies }\end{array}$ & $\begin{array}{l}\text { PEG }(n=363) \\
\text { lactulose }(n=364)\end{array}$ & & $\begin{array}{l}\text { Mean stools/week: MD=0.65 } \\
\text { favoured PEG ( } 5 \text { high quality } \\
\text { studies) } \\
\text { Bristol Stool Score: MD=0.89 } \\
\text { favoured PEG ( } 2 \text { studies) } \\
\text { Relief of abdominal pain: } \\
\text { MD=2.09 favoured lactulose } \\
\text { ( } 3 \text { studies) } \\
\text { Use of additional products: } \\
\text { MD=4.00 favoured PEG } \\
\text { (3 studies) }\end{array}$ & $\begin{array}{l}\text { Subgroup analysis according to age } \\
\text { (children and adults) had MDs that } \\
\text { favoured PEG for mean stools/week } \\
\text { and use of additional products for both; } \\
\text { the MD for Bristol Stool Score } \\
\text { (insufficient data for adults) and relief } \\
\text { of abdominal pain favoured PEG for } \\
\text { children }\end{array}$ \\
\hline
\end{tabular}


TABLE 2 - CONTINUED

Summary of studies examining the efficacy of nonstimulant laxatives

\begin{tabular}{|c|c|c|c|c|c|}
\hline $\begin{array}{l}\text { Author (ref), } \\
\text { year }\end{array}$ & Design/duration & $\begin{array}{l}\text { Treatment (n, mean } \\
\text { age) }\end{array}$ & $\begin{array}{l}\text { Comparator ( } \mathrm{n} \text {, mean } \\
\text { age) }\end{array}$ & Measures & Outcomes \\
\hline $\begin{array}{l}\text { Loening- } \\
\text { Baucke and } \\
\text { Pashankar } \\
\text { (43), 2006, } \\
\text { Rome III }\end{array}$ & $\begin{array}{l}\text { Randomized } \\
\text { comparative trial } \\
12 \text { months }\end{array}$ & $\begin{array}{c}\text { PEG }(0.7 \mathrm{~g} / \mathrm{kg} / \text { day }) \\
(\mathrm{n}=39,8.0 \text { years })\end{array}$ & $\begin{array}{c}\text { MOM }(2 \mathrm{~mL} / \mathrm{kg} / \text { day }) \\
(\mathrm{n}=40,8.2 \text { years })\end{array}$ & $\begin{array}{l}\text { Mean BM/week, decrease in } \\
\text { abdominal pain and fecal } \\
\text { incontinence }\end{array}$ & $\begin{array}{l}\text { PEG ( } 3.5 \text { to } 6.8 \text { ) vs MOM ( } 3.5 \text { to } 8.2 \text { ). } \\
\text { NSD found between treatments } \\
\text { regarding improvement and recovery } \\
\text { rates }\end{array}$ \\
\hline $\begin{array}{l}\text { Ratanamong- } \\
\text { kol et al (44), }\end{array}$ & $\mathrm{RCT}$ & $\begin{array}{l}\text { PEG }(n=46, \\
1-4 \text { years) }\end{array}$ & $\begin{array}{r}\text { MOM }(n=43 \\
1-4 \text { years) }\end{array}$ & "Improvement" & PEG > MOM (91\% vs $65 \%) ; P=0.003$ \\
\hline $\begin{array}{l}\text { 2009, Rome } \\
\text { III }\end{array}$ & 4 weeks & & & Compliance rate & PEG > MOM (89\% vs $72 \%) ; P=0.041$ \\
\hline \multirow[t]{3}{*}{$\begin{array}{l}\text { Gomes et al } \\
\text { (45), 2011, } \\
\text { Rome III }\end{array}$} & $\mathrm{RCT}$ & $\begin{array}{l}\text { PEG }(0.5-1.5 \mathrm{~g} / \mathrm{kg} / \\
\text { day })(\mathrm{n}=17,4.3 \\
\text { years })\end{array}$ & $\begin{array}{l}\text { MOM }(1-3 \mathrm{~mL} / \mathrm{kg} / \text { day }) \\
\quad(n=21,5.1 \text { years })\end{array}$ & Mean BM/week & NSD \\
\hline & 6 months & & & Bristol Stool Score & NSD \\
\hline & & & & Subjective scores & $\begin{array}{l}\text { NSD for pain, abdominal pain, straining } \\
\text { and incontinence }\end{array}$ \\
\hline \multirow[t]{2}{*}{$\begin{array}{l}\text { Wang et al } \\
(46), 2004\end{array}$} & $\begin{array}{c}\text { Randomized } \\
\text { open-label }\end{array}$ & $\begin{array}{l}P E G+E 13.8 \mathrm{~g} \times \\
2 \text { days }(\mathrm{n}=63 \\
51 \text { years })\end{array}$ & $\begin{array}{l}\text { Psyllium } 3.5 \mathrm{~g} \times \\
2 \text { days }(\mathrm{n}=63 \\
50 \text { years })\end{array}$ & Mean BM/week & $\begin{array}{l}\text { Both groups increased BM/week but } \\
\text { efficacy was higher with PEG+E } \\
(92 \% \text { vs } 73 \%) ; P=0.005\end{array}$ \\
\hline & 14 day & & & Normalized stools & PEG+E $87.3 \%$ vs $66.7 \% ; P<0.001$ \\
\hline
\end{tabular}

BM Bowel movements; MD Mean difference, MOM Milk of magnesia; n/a Not available or mentioned in the manuscript; NNT Number needed to treat; NSD No significant difference; PEG Polyethylene glycol; PEG+E Polyethylene glycol with electrolytes; RCT Randomized controlled trial; ref Reference; RR Risk ratio; vs Versus; WMD Weighted mean difference

Similar to the PEG versus placebo studies, these PEG versus lactulose studies varied considerably with respect to the definition of constipation used to select patients, ages and study duration, but not with respect to study design because all were RCTs. In spite of these variations, the individual trials and pooled data analyses consistently indicated that PEG was significantly better than lactulose to relieve both objective and subjective measures of functional constipation.

PEG compared with milk of magnesia: Each of these RCTs used the Rome III or similar definition as an integral part of their selection criteria of pediatric patients with functional constipation (43-45). The earliest study by Loening-Baucke and Pahankar (43) ( $n=79)$ found no significant difference between PEG or milk of magnesia cohorts with respect to overall improvement of constipation and recovery rates. Similarly, the 2011 study by Gomes et al (45) ( $n=38)$ reported no significant difference between the two cohorts with respect to the mean number of bowel movements per week, Bristol stool score and subjective scores (including pain, straining, and incontinence). Conversely, in the 2009 study by Ratanamongkol et al (44) $(n=89)$, the cohort receiving PEG experienced significantly greater improvements in functional constipation symptoms. Each of the three studies found that the patients preferred PEG over milk of magnesia.

Interestingly, the two studies that reported no significant differences between experimental measures of the efficacy of PEG and milk of magnesia were conducted for long periods, over six and 12 months, respectively, and enrolled older children $(43,45)$. Instead, the clinical trial by Ratanamongkol et al (44) lasted only four weeks and recruited younger children (one to four years of age).

Regardless of the differences in study design, the 2012 meta-analysis by Gordon et al (33) pooled data from three studies (211 patients) and found that the mean difference with respect to the mean number of stools per week was 0.69 and favoured PEG over milk of magnesia.

PEG compared with bulking agents: Only one study that compared PEG versus psyllium, a bulking agent, was retrieved (46). In this randomized, but open-label, trial lasting 14 days, adults (mean age 51 years) were recruited providing that they had $\leq 2$ bowel movements per week and a mean Bristol stool score of 1 to 3 for $>3$ months. The 126 patients were randomly assigned to either PEG or psyllium $(3.5 \mathrm{~g}$ twice per day) treatment group. At 14 days, the mean weekly number of bowel movements increased from 1.18 at baseline to 8.48 in the
PEG group, and 1.33 to 5.71 in the psyllium group $(\mathrm{P}<0.001)$. Before the study, none of the patients had normal stool forms. By 14 days, $87.3 \%$ of the PEG group, compared with $66.7 \%$ in the psyllium group, had normal stools $(\mathrm{P}<0.001)$. There were no significant differences identified with rates of improvement for flatulence, abdominal pain, straining, pain or passing gas. Furthermore, no significant differences were found with respect to treatment side effects. Dizziness was reported by three patients in the PEG group and dry mouth was reported by three in the psyllium group. Overall efficacy was a combined score taking into account weekly bowel movement frequency, stool formation and difficulties associated with bowel movements. Patients taking PEG scored $92.1 \%$ while those taking psyllium scored 73.0\% $(\mathrm{P}=0.005)$.

\section{Stimulant laxatives}

Two clinical trials and one meta-analysis (Table 3) examining the efficacy of stimulant laxatives (bisacodyl and liquid sodium picosulphate) for the treatment of constipation were retrieved.

Sodium picosulphate and bisacodyl: Because sodium picosulphate and bisacodyl are both prodrugs that are converted into the active form of bis-(p-hydroxyphenyl)-pyridyl-2-methane in the gut, it is reasonable to consider these two laxatives as being similar. In 2010, Mueller-Lissner et al (47) conducted a double-blinded RCT at 45 study sites in Germany. Following a two-week period, in which no laxatives were permitted, only individuals who fulfilled Rome III criteria for functional constipation were allocated $(n=367) 2: 1$ to receive either sodium picosulphate drops ( $10 \mathrm{mg} /$ day) or placebo for four weeks. The mean number of complete spontaneous bowel movements (CSBMs) per week increased from 0.9 to 32.4 in the sodium picosulphate group compared with an increase from 1.1 to 1.7 in the placebo group $(\mathrm{P}<0.0001)$. Results from the Short-Form-36v2 quality of life survey indicated that sodium picosulphate treatment led to significantly greater score improvements from baseline in two of the eight components being general health $(\mathrm{P}=0.008)$ and mental components $(\mathrm{P}=0.048)$ relative to placebo. The Patient Assessment of Constipation Quality of Life (PAC-QOL) results found that patients in the treatment group experienced significant improvements overall, and in each of the four subcomponents compared with the placebo group $(\mathrm{P}<0.001)$. In the sodium picosulphate group, $31.8 \%$ of patients 
TABLE 3

Summary of studies assessing the efficacy of stimulant laxatives compared with placebo

\begin{tabular}{|c|c|c|c|c|c|}
\hline $\begin{array}{l}\text { Author } \\
\text { (reference), } \\
\text { year }\end{array}$ & Design (duration) & Treatment & Placebo & Outcomes & Comments \\
\hline $\begin{array}{l}\text { Mueller-Lissner } \\
\text { et al (47), } \\
\text { 2010, Rome } \\
\text { III }\end{array}$ & $\begin{array}{l}\text { Double-blinded } \\
\text { RCT at } 43 \text { study } \\
\text { sites (4 weeks) }\end{array}$ & $\begin{array}{l}\text { SPS, } 10 \mathrm{mg} / \text { day } \\
\text { (or } 18 \text { drops) } \\
\text { ( } \mathrm{n}=233, \text { mean } \\
\text { age } 50.2 \text { years) }\end{array}$ & $\begin{array}{l}\text { Placebo }(n=134, \\
\text { mean age } \\
51.9 \text { years })\end{array}$ & $\begin{array}{l}\text { Final mean }( \pm \text { SD }) \text { CSBMs: SPS } 3.4 \pm 0.2 \\
\text { versus placebo } 1.7 \pm 0 \text {. Greater } \\
\text { improvement for SPS for } \triangle \text { CSBM, } \\
\geq 3 \text { CSBMs/week, } \geq 1 \text { CSBM/day } \\
(P<0.0001) \text {. Final PAC-QOL, } 87.7 \% \text { of } \\
\text { SPS versus } 45.8 \% \text { of placebo rated } \\
\text { efficacy as either good or satisfactory } \\
(P<0.0001) \text {. }\end{array}$ & $\begin{array}{l}\text { SPS dose titrated down by } 50 \% \text { at end } \\
\text { of study. Diarrhea affected } 31.8 \% \text { of } \\
\text { SPS versus } 4.5 \% \text { of placebo patients. } \\
\text { Abdominal pain affected } 5.6 \% \text { of SPS } \\
\text { versus } 2.2 \% \text { of placebo patients }\end{array}$ \\
\hline $\begin{array}{l}\text { Kamm et al } \\
\text { (48), 2011, } \\
\text { Rome III }\end{array}$ & $\begin{array}{l}\text { Double-blinded } \\
\text { RCT, } 27 \text { centres } \\
\text { (4 weeks) }\end{array}$ & $\begin{array}{l}\text { Bisacodyl } \\
\text { (Dulcolax*) } \\
10 \mathrm{mg} / \text { day } \\
\text { (n=191, mean } \\
\text { age } 55.8 \text { years })\end{array}$ & $\begin{array}{l}\text { Placebo }(n=106, \\
\text { mean age } \\
54.7 \text { years })\end{array}$ & $\begin{array}{l}\text { Final mean }( \pm \mathrm{SD}) \text { CSBMs: bisacodyl } \\
5.2 \pm 0.3 \text { versus placebo } 1.9 \pm 0.3 \text {. Greater } \\
\text { improvement for bisacodyl in } \triangle \mathrm{CSBMs} \\
(\mathrm{P}<0.0001) \text {. PAC-QOL } \\
\text { bisacodyl improvement greater than } \\
\text { placebo }(P \leq 0.007) \text {. }\end{array}$ & $\begin{array}{l}\text { All secondary end points demonstrated } \\
\text { efficacy for bisacodyl }(P<0.0001) \text {. } \\
\text { Bisacodyl had } 44 \mathrm{AEs} \text { versus } 6 \mathrm{AEs} \\
\text { with placebo, leading to discontinuation. } \\
\text { The most common bisacodyl AE was } \\
\text { diarrhea occurring in } 53.4 \% \text { of patients }\end{array}$ \\
\hline
\end{tabular}

*Boehrigner Ingelheim Pharmaceuticals, USA. AE Adverse event; CSBM Complete spontaneous bowel movement; NNH Number needed to harm; NNT Number needed to treat; PAC-QOL Patient Assessment - Quality of Life questionnaire: RCT Randomized controlled trial; SPS Sodium picosulfate

TABLE 4

Summary of a clinical trial comparing nonstimulant versus stimulant laxatives

\begin{tabular}{|c|c|c|c|c|c|}
\hline \multirow{2}{*}{$\begin{array}{l}\text { Author } \\
\text { (reference), year }\end{array}$} & \multirow[b]{2}{*}{ Design/duration } & \multicolumn{2}{|c|}{ Laxative } & \multirow[b]{2}{*}{ Outcomes } & \multirow[b]{2}{*}{ Comments } \\
\hline & & Nonstimulant $(n=20)$ & Stimulant $(n=20)$ & & \\
\hline Perkin (49), 1977 & $\begin{array}{l}\text { Cross-over trial: } 1 \text { week } \rightarrow \\
1 \text { week washout } \rightarrow \\
1 \text { week treatment }\end{array}$ & $\begin{array}{l}\text { Lactulose }(10-15 \mathrm{~mL} / \text { day }) \\
\quad(<15 \text { years })\end{array}$ & $\begin{array}{l}\text { Senna syrup }(10-20 \mathrm{~mL} / \\
\text { day) (<15 years) }\end{array}$ & $\begin{array}{l}\text { Lactulose had more normal } \\
\text { stools }(P<0.01) \text { and fewer } \\
\text { side effects }(P<0.001)\end{array}$ & $\begin{array}{l}\text { Adverse events: lactulose } \\
\qquad(n=1) ; \text { senna syrup }(n=30)\end{array}$ \\
\hline
\end{tabular}

experienced diarrhea and $5.6 \%$ experienced abdominal pain in contrast with $4.5 \%$ and $2.2 \%$ in the placebo group.

A double-blind RCT, conducted at 27 centres in the United Kingdom, compared the efficacy of bisacodyl (Dulcolax [Boehrigner Ingelheim, USA], $10 \mathrm{mg} /$ day, $\mathrm{n}=247$ ) versus placebo ( $\mathrm{n}=121)$ (48). In this four-week trial, adults (mean age 55 years) fulfilling Rome III criteria for functional constipation were assessed for CSBMs and secondary measures including the PAC-QOL. The mean number of CSBMs per week increased from a baseline of 1.1 for both groups to 5.2 in the bisacodyl group and 1.9 in the placebo $(\mathrm{P}<0.0001)$. Secondary measures (total CSBMs per week, spontaneous bowel movements and constipation symptoms) were significantly improved in the bisacodyl group compared with placebo ( $\mathrm{P}<0.0001$ for each measure). The PACQOL score and subscales (satisfaction, psychosocial and physical discomforts, worries and concerns) significantly improved compared with baseline in the bisacodyl group versus placebo $(\mathrm{P} \leq 0.007)$. In total, bisacodyl treatment led to 44 adverse events versus six with placebo, which led to study discontinuation; the most common adverse event was diarrhea.

In the meta-analysis by Ford et al (36), there were $42.1 \%$ nonresponders in the stimulant-treatment group compared with $78.0 \%$ in the placebo group using a pooled patient population of 735 . The number needed to treat was determined to be 3 (95\% CI 2 to 3.5 ) as was the number needed to harm (3 [95\% CI 2 to 6$]$ ).

Nonstimulant laxatives compared with stimulant laxatives A 1977 crossover trial $(n=20)$, entailing a one-week treatment period, one-week washout then another one-week treatment (49), compared lactulose $(10 \mathrm{~mL} /$ day to $15 \mathrm{~mL} /$ day $)$ versus senna syrup $(10 \mathrm{~mL} /$ day to
$20 \mathrm{~mL} /$ day) for the treatment of pediatric constipation (Table 4). Patients $(<15$ years of age) were randomly assigned to a one-week treatment group on entry into the trial. The mean number of stools per week was 6.4 and 6.0 for lactulose and senna treatment, respectively. The number of patients who had normal stools per day (classified according to loose, normal, hard or none) was significantly higher during the lactulose treatment week compared with senna (4.6 versus 3.0; $\mathrm{P}<0.01$ ). With respect to side effects, significantly fewer were reported during the lactulose treatment compared with senna (one versus 30; $\mathrm{P}<0.001$ ). The most common side effects were colic followed by diarrhea.

\section{DISCUSSION}

It is well recognized that the evidence to date, regarding the efficacy of laxatives for the treatment of functional constipation, is associated with key limitations, the first being the wide variety of definitions for functional constipation and its impact on trial patient selection and study end points. The Rome III definition is an aid to discriminate between perceived and functional constipation while recognizing the substantial biological variability regarding the frequency of normal bowel movements. Given that functional constipation is a longstanding condition, it is reasonable that clinical trials should be conducted for longer durations ( $>6$ months) rather than days or weeks to comprehensively evaluate the efficacy of the laxative treatment for this patient population. Furthermore, the absence of quality of life measures is a large unknown variable in the evaluation of laxatives, primarily nonstimulants, because the day-to-day impact of possible side effects or benefits have not been captured.

Despite these limitations, there is considerable evidence from quality studies that both stimulant and nonstimulant laxatives are more 
effective than placebo. Specifically, osmotic laxatives are more effective than placebo, with PEG being the most effective. Taking this one step further, PEG without electrolytes is preferable over PEG with electrolytes based on palatability, which will ultimately affect patient compliance. It is interesting to note that in clinical practice, docusate salts are still commonly used although the evidence base is nominal compared with that for PEG.

Unlike the evidence base for nonstimulant laxatives, it is significantly smaller for stimulants. However, the two studies assessing the efficacy of sodium picosulphate and bisacodyl used the Rome III criteria for functional constipation and included quality of life assessments, thereby enhancing the quality of the study results $(47,48)$. Supported by a meta-analysis, it is clear that both stimulants are significantly better than placebo for the resolution of functional constipation (36). It should be noted that diarrhea is a common adverse event with stimulant laxatives. The advantage of using liquid sodium picosulphate is that treatment can be reduced in small amounts from the standard $10 \mathrm{mg} /$ day or 18 drops to a lower dose to prevent diarrhea while still benefiting from functional constipation relief.

\section{REFERENCES}

1. Whorton JC. Inner Hygiene: Constipation and the Pursuit of Health in Modern Society. New York: Oxford University Press, 2000:1-315

2. Connell AM, Hilton C, Irvine G, Lennard-Jones JE, Misiewicz JJ. Variation of bowel habit in two population samples. BM] 1965;2:1095-9.

3. Heaton KW, Radvan J, Cripps H, Mountford RA, Braddon FE, Hughes AO. Defecation frequency and timing, and stool form in the general population: A prospective study. Gut 1992;33:818-24.

4. Longstreth GF, Thompson WG, Chey WD, Houghton LA, Mearin F, Spiller RC. Functional bowel disorders. Gastroenterology 2006;130:1480-91.

5. Thompson WG, Longstreth GF, Drossman DA, Heaton KW, Irvine EJ, Muller-Lissner SA. Functional bowel disorders and functional abdominal pain. Gut 1999;45:II43-II47.

6. Ferrazzi S, Thompson WG, Irvine EJ, Pare P, Rance L. Diagnosis of constipation in family practice. Can J Gastroenterol 2002;16:159-64.

7. Pare P, Ferrazzi S, Thompson WG, Irvine EJ, Rance L. An epidemiological survey of constipation in Canada: Definitions, rates, demographics, and predictors of health care seeking. Am J Gastroenterol 2001;96:3130-7.

8. Talley NJ, Weaver AL, Zinsmeister AR, Melton LJ III. Functional constipation, and outlet delay. Gastroenterology 1993;105:781-90.

9. Euromonitor International. GMID - Global Market Information Database. < http://www.euromonitor.com> (Accessed June 6, 2014).

10. Chang FY, Chen PH, Wu TC, et al. Prevalence of functional gastrointestinal disorders in Taiwan: Questionnaire-based survey for adults based on the Rome III criteria. Asia Pac J Clin Nutr 2012;21:594-600.

11. Breckan RK, Asfeldt AM, Straume B, Florholmen J, Paulssen EJ. Prevalence, comorbidity, and risk factors for functional bowel symptoms: A population-based survey in northern Norway. Scand J Gastroenterol 2012;47:1274-82.

12. Sanchez MI, Bercik P. Epidemiology and burden of chronic constipation. Can J Gastroenterol 2011;(25 Suppl B):11B-5B.

13. Johanson JF. Geographic distribution of constipation in the United States. Am J Gastroenterol 1998;93:188-91.

14. Maspero G. Ebers Papyrus. Etudes de mythologie et d'achéologie égyptiennes. Paris, 1898.

15. World Gastroenterology Organisation Global Guidelines. Constipation: A global perspective. < www.worldgastroenterology. org/assets/export/userfiles/05_constipation.pdf> (Accessed June 6, 2014).

16. Rutter K, Maxwell D. Diseases of the alimentary system. Constipation and laxative abuse. BMJ 1976;2:997-1000.

17. Smith B. Effect of irritant purgatives on the myenteric plexus in man and the mouse. Gut 1968;9:139-43.

18. Smith B. Pathologic changes in the colon produced by anthraquinone purgatives. Dis Colon Rectum 1973;16:455-8.

19. Dufour P, Gendre P. Ultrastructure of mouse intestinal mucosa and changes observed after long term anthraquinone administration. Gut 1984;25:1358-63.
Although much emphasis has been placed on the assessment of nonstimulant laxatives, comparative efficacy trials with stimulant laxatives are appreciably lacking. The single study retrieved from 1977 used questionable patient-selection criteria (children had to have a history of constipation treated at home for at least three months) and included no baseline measures of bowel movement frequency to correlate the 1977 interpretation of 'chronic constipation' with the Rome III criteria. The results presented indicate that although the two laxative treatments led to a similar bowel movement frequency per day, patients taking lactulose had significantly more normal stools and fewer side effects with respect to diarrhea and abdominal distention.

Although progress has been made to provide quality evidence supporting the efficacy of laxatives for the treatment of functional constipation, it is obvious that further studies are needed to assess the impact of long-term treatment and comparisons of efficacy among laxatives, particularly between nonstimulants and stimulants.

DISCLOSURES: The authors have no financial disclosures or conflicts of interest to declare.

20. Bharucha AE, Pemberton JH, Locke GRI. American Gastroenterological Association technical review on constipation. Gastroenterology 2013;144:218-38.

21. Kiernan JA, Heinicke EA. Sennosides do not kill myenteric neurons in the colon of the rat or mouse. Neuroscience 1989;30:837-42.

22. McRorie JW, Daggy BP, Morel JG, Diersing PS, Miner PB, Robinson M. Psyllium is superior to docusate sodium for treatment of chronic constipation. Aliment Pharmacol Ther 1998;12:491-7.

23. Urganci N, Akyildiz B, Polat TB. A comparative study: The efficacy of liquid paraffin and lactulose in management of chronic functional constipation. Pediatr Int 2005;47:15-9.

24. Rafati M, Karami H, Salehifar E, Karimzadeh A. Clinical efficacy and safety of polyethylene glycol 3350 versus liquid paraffin in the treatment of pediatric functional constipation. Daru 2011;19:154-8.

25. Cleveland MV, Flavin DP, Ruben RA, Epstein RM, Clark GE. New polyethylene glycol laxative for treatment of constipation in adults: A randomized, double-blind, placebo-controlled study. South Med J 2001;94:478-81.

26. DiPalma JA, DeRidder PH, Orlando RC, Kolts BE, Cleveland MB. A randomized, placebo-controlled, multicenter study of the safety and efficacy of a new polyethylene glycol laxative. Am J Gastroenterol 2000;95:446-50.

27. DiPalma JA, Cleveland MV, McGowan J, Herrera JL. A randomized, multicenter, placebo-controlled trial of polyethylene glycol laxative for chronic treatment of chronic constipation. Am J Gastroenterol 2007;102:1436-41.

28. Nurko S, Youssef NN, Sabri M, et al. PEG3350 in the treatment of childhood constipation: A multicenter, double-blinded, placebo-controlled trial. J Pediatr 2008;153:254-61, 261.

29. Andorsky RI, Goldner F. Colonic lavage solution (polyethylene glycol electrolyte lavage solution) as a treatment for chronic constipation: A double-blind, placebo-controlled study. Am J Gastroenterol 1990;85:261-5.

30. Corazziari E, Badiali D, Habib FI, et al. Small volume isosmotic polyethylene glycol electrolyte balanced solution (PMF-100) in treatment of chronic nonorganic constipation. Dig Dis Sci 1996;41:1636-42.

31. Thomson MA, Jenkins HR, Bisset WM, et al. Polyethylene glycol 3350 plus electrolytes for chronic constipation in children: A double blind, placebo controlled, crossover study. Arch Dis Child 2007;92:996-1000.

32. Belsey JD, Geraint M, Dixon TA. Systematic review and meta analysis: Polyethylene glycol in adults with non-organic constipation. Int J Clin Pract 2010;64:944-55.

33. Gordon M, Naidoo K, Akobeng AK, Thomas AG. Osmotic and stimulant laxatives for the management of childhood constipation. Cochrane Database Syst Rev 2012;7:CD009118.

34. Chaussade S, Minic M. Comparison of efficacy and safety of two doses of two different polyethylene glycol-based laxatives in the treatment of constipation. Aliment Pharmacol Ther 2003;17:165-72. 
35. Savino F, Viola S, Erasmo M, Di Nardo G, Oliva S, Cucchiara S. Efficacy and tolerability of peg-only laxative on faecal impaction and chronic constipation in children. A controlled double blind randomized study vs a standard peg-electrolyte laxative. BMC Pediatr 2012;12:178.

36. Ford AC, Suares NC. Effect of laxatives and pharmacological therapies in chronic idiopathic constipation: Systematic review and meta-analysis. Gut 2011;60:209-18.

37. Bouhnik Y, Neut C, Raskine L, et al. Prospective, randomized, parallel-group trial to evaluate the effects of lactulose and polyethylene glycol-4000 on colonic flora in chronic idiopathic constipation. Aliment Pharmacol Ther 2004;19:889-99.

38. Dupont C, Leluyer B, Maamri N, et al. Double-blind randomized evaluation of clinical and biological tolerance of polyethylene glycol 4000 versus lactulose in constipated children. J Pediatr Gastroenterol Nutr 2005;41:625-33.

39. Attar A, Lemann M, Ferguson A, et al. Comparison of a low dose polyethylene glycol electrolyte solution with lactulose for treatment of chronic constipation. Gut 1999;44:226-30.

40. Candy DC, Edwards D, Geraint M. Treatment of faecal impaction with polyethelene glycol plus electrolytes $(\mathrm{PGE}+\mathrm{E})$ followed by a double-blind comparison of PEG + E versus lactulose as maintenance therapy. J Pediatr Gastroenterol Nutr 2006;43:65-70.

41. Voskuijl W, de LF, Verwijs W, et al. PEG 3350 (Transipeg) versus lactulose in the treatment of childhood functional constipation: A double blind, randomised, controlled, multicentre trial. Gut 2004;53:1590-4.

42. Lee-Robichaud H, Thomas K, Morgan J, Nelson RL. Lactulose versus polyethylene glycol for chronic constipation. Cochrane Database Syst Rev 2011CD007570.
43. Loening-Baucke V, Pashankar DS. A randomized, prospective, comparison study of polyethylene glycol 3350 without electrolytes and milk of magnesia for children with constipation and fecal incontinence. Pediatrics 2006;118:528-35.

44. Ratanamongkol P, Lertmaharit S, Jongpiputvanich S. Polyethylene glycol 4000 without electrolytes versus milk of magnesia for the treatment of Functional constipation in infants and young children: A randomized controlled trial. Asian Biomed 2009;3:391-9.

45. Gomes PB, Duarte MA, Melo Mdo C. Comparison of the effectiveness of polyethylene glycol 4000 without electrolytes and magnesium hydroxide in the treatment of chronic functional constipation in children. J Pediatr (Rio J) 2011;87:24-8.

46. Wang HJ, Liang XM, Yu ZL, Zhou LY, Lin SR, Geraint M. A randomised, controlled comparison of low-dose polyethylene glycol 3350 plus electrolytes with Ispaghula husk in the treatment of adults with chronic functional constipation. Clin Drug Investig 2004;24:569-76.

47. Mueller-Lissner S, Kamm MA, Wald A et al. Multicenter, 4-week, double-blind, randomized, placebo-controlled trial of sodium picosulfate in patients with chronic constipation. Am J Gastroenterol 2010;105:897-903.

48. Kamm MA, Mueller-Lissner S, Wald A, Richter E, Swallow R, Gessner U. Oral bisacodyl is effective and well-tolerated in patients with chronic constipation. Clin Gastroenterol Hepatol 2011;9:577-83.

49. Perkin JM. Constipation in childhood: A controlled comparison between lactulose and standardized senna. Curr Med Res Opin $1977 ; 4: 540-3$ 


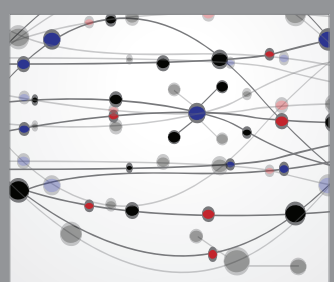

The Scientific World Journal
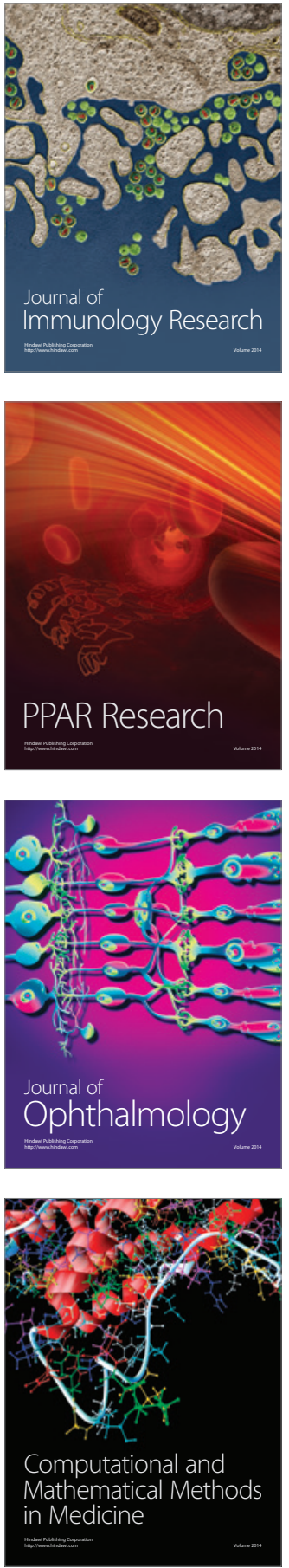

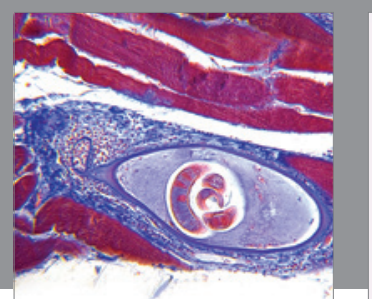

Gastroenterology Research and Practice

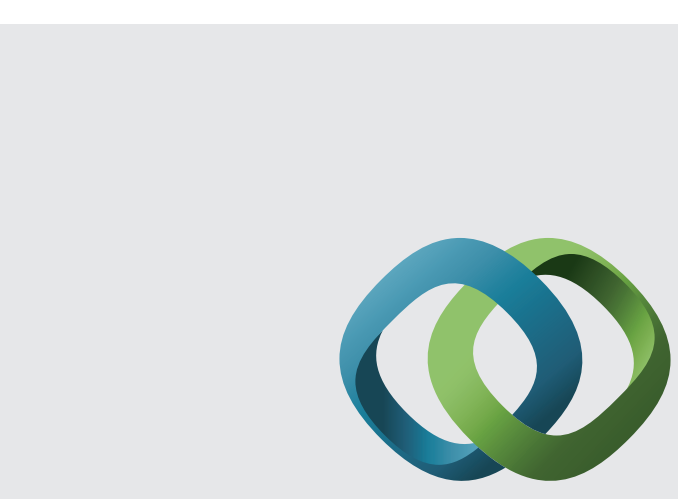

\section{Hindawi}

Submit your manuscripts at

http://www.hindawi.com
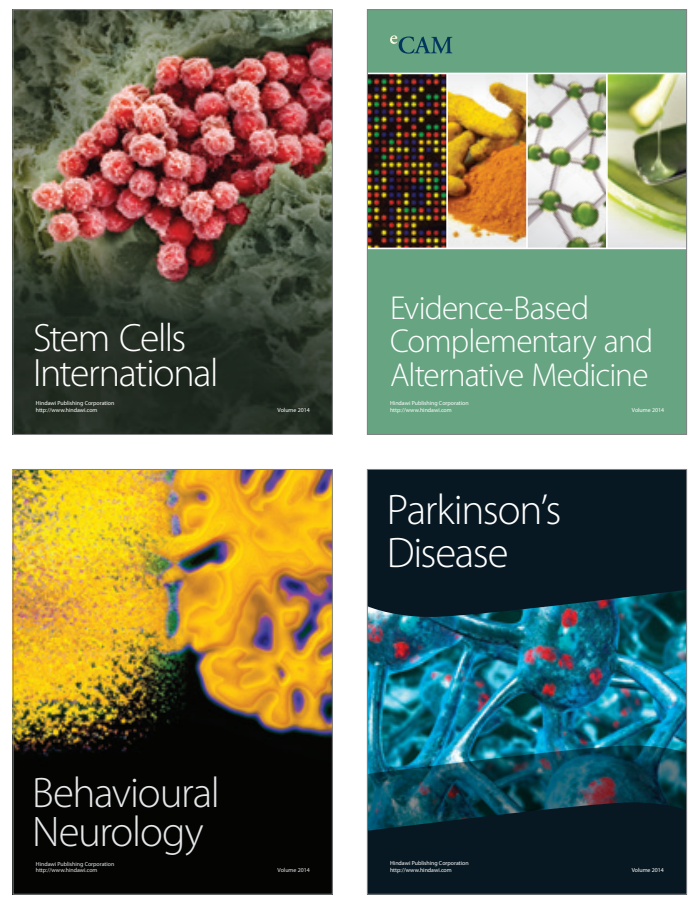
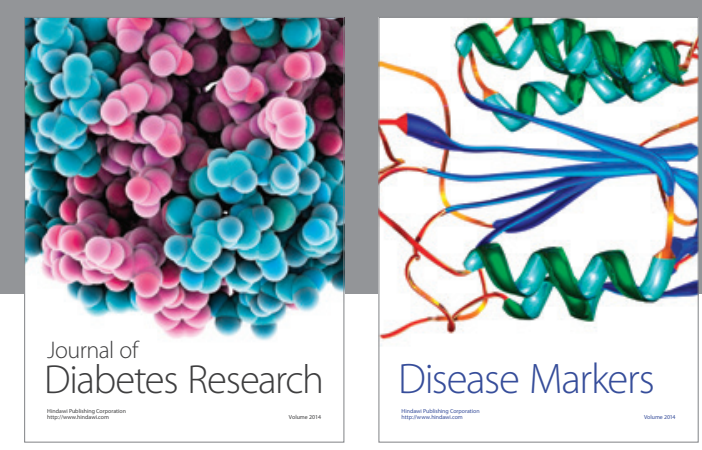

Disease Markers
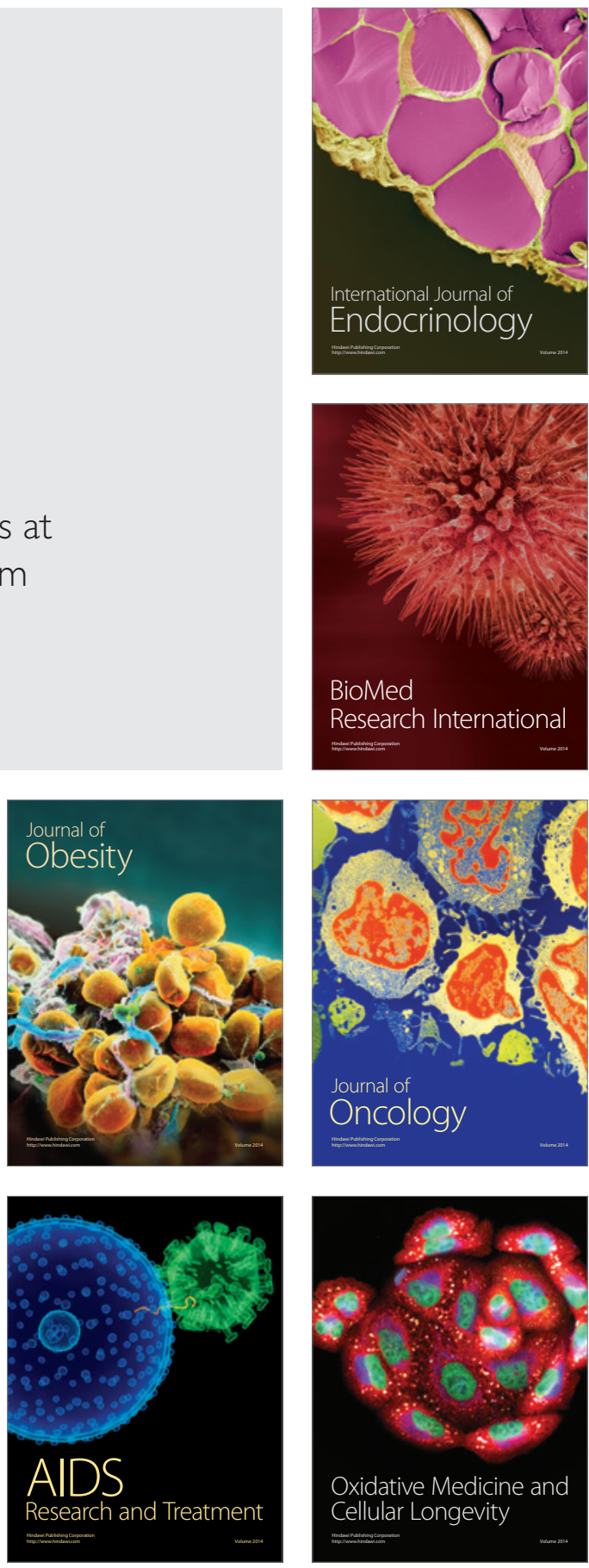